\title{
Simple Spectrophotometric Method for Determination of Paroxetine in Tablets Using 1,2-Naphthoquinone-4-Sulphonate as a Chromogenic Reagent
}

\author{
Ibrahim A. Darwish, Heba H. Abdine, Sawsan M. Amer, and Lama I. Al-Rayes \\ Department of Pharmaceutical Chemistry, College of Pharmacy, King Saud University, P.O. Box 2457, \\ Riyadh 11451, Saudi Arabia \\ Correspondence should be addressed to Ibrahim A. Darwish, idarwish@ksu.edu.sa
}

Received 13 October 2008; Accepted 10 February 2009

Recommended by Maria Augusta Raggi

\begin{abstract}
Simple and rapid spectrophotometric method has been developed and validated for the determination of paroxetine (PRX) in tablets. The proposed method was based on nucleophilic substitution reaction of PRX with 1,2-naphthoquinone-4-sulphonate (NQS) in an alkaline medium to form an orange-colored product of maximum absorption peak $\left(\lambda_{\max }\right)$ at $488 \mathrm{~nm}$. The stoichiometry and kinetics of the reaction were studied, and the reaction mechanism was postulated. Under the optimized reaction conditions, Beer's law correlating the absorbance (A) with PRX concentration (C) was obeyed in the range of $1-8 \mu \mathrm{g} \mathrm{mL} \mathrm{m}^{-1}$. The regression equation for the calibration data was: $\mathrm{A}=0.0031+0.1609 \mathrm{C}$, with good correlation coefficients $(0.9992)$. The molar absorptivity $(\varepsilon)$ was $5.9 \times 10^{5} \mathrm{~L} \mathrm{~mol}^{-1} 1 \mathrm{~cm}^{-1}$. The limits of detection and quantitation were 0.3 and $0.8 \mu \mathrm{g} \mathrm{mL} \mathrm{m}^{-1}$, respectively. The precision of the method was satisfactory; the values of relative standard deviations did not exceed $2 \%$. The proposed method was successfully applied to the determination of PRX in its pharmaceutical tablets with good accuracy and precisions; the label claim percentage was $97.17 \pm 1.06 \%$. The results obtained by the proposed method were comparable with those obtained by the official method.
\end{abstract}

Copyright () 2009 Ibrahim A. Darwish et al. This is an open access article distributed under the Creative Commons Attribution License, which permits unrestricted use, distribution, and reproduction in any medium, provided the original work is properly cited.

\section{Introduction}

Paroxetine; (3S,4R)-3-[(1,3-benzodioxol-5-vloxy)methyl]4-(4-flurophenyl) piperidine (PRX) is a new generation antidepressant drug. It exerts its antidepressant effect through a selective inhibition for the reuptake of the neurotransmitter serotonin by the presynaptic receptors. PRX is comparable to the tricyclic antidepressants in their clinical efficacy, however, PRX is safer and has greater acceptance by the patients [1]. It is also prescribed in the treatment of related disorders, such as obsessive-compulsive disorder, panic fits, social phobia, and posttraumatic stress [2]. PRX is devoid of sedative effect and remarkably safe in overdose. PRX takes 5.2 hours to reach the peak, with extended half-life ( 21 hours) that allowed the introduction of formulations for once-daily dosing [3].
These combined qualities made PRX the most widely prescribed antidepressants [4].

The methods reported for quantitative determination of PRX in tablets and/or biological fluids include voltammetry $[5,6]$, densitometry $[7,8]$, high-performance liquid chromatography [9-14], gas chromatography [15-17], and capillary electrophoresis [18]. These methods offered the required sensitivity and selectivity for the analysis of PRX in biological fluids; however, their sophisticated instrumentation and high analysis cost limited their routine use in quality control laboratories for analysis of PRX in its pharmaceutical tablets.

Spectrophotometry is considered the most convenient analytical technique because of its inherent simplicity, low cost, and wide availability in most quality control laboratories. However, few spectrophotometric methods have been reported for its determination in tablets [19-21]. These 
methods were based on formation of ion-pair associates with bromophenol blue, bromothymol blue, and bromocresol green [19], formation of charge-transfer complexes with 7,7,8,8-tetracyanoquinodimethane and chloranilic acid [20], formation of vinylamino-substituted haloquinone derivatives with haloquinone reagents in presence of acetaldehyde [21], and formation of condensation product with 7-chloro4-nitrobenzofurazon [19]. These methods were associated with some major drawbacks such as laborious multiple extraction steps in the analysis by ion pair formation-based methods [19], and in preparation of the samples for the analysis by the methods relied on PRX base, rather than the hydrochloride salt $[20,21]$. Furthermore, the analytical reactions were long and thus the procedures were timeconsuming $[19,21]$. For these reasons, the development of new alternative simple and rapid spectrophotometric method for the determination of PRX in its tablets was very essential.

1,2-naphthoquinone-4-sulphonic sulphonate (NQS) has been used as a chromogenic reagent for the spectrophotometric determination of many pharmaceutical amines [22-26]. However, the reaction between NQS and PRX has not been investigated so far. The present study describes the evaluation of NQS as a chromogenic reagent in the development of simple and rapid spectrophotometric method for the determination of PRX in its tablets.

\section{Experimental}

2.1. Apparatus. Double beam V-530 (JASCO Co. Ltd., Kyoto, Japan) ultraviolet-visible spectrophotometer with matched $1 \mathrm{~cm}$ quartz cells was used for all the spectrophotometric measurements. pH meter, Model 350 (Bibby Scientific Ltd., T/As Jenway, Essex, UK).

2.2. Reagents and Materials. Paroxetine hydrochloride (PRX; SmithKline Beecham Pharmaceuticals, Bentford, England) was obtained and used as received; its purity was $99.8 \pm$ $1.45 \%$. A solution of $0.5 \%(\mathrm{w} / \mathrm{v})$ of 1,2 -naphthoquinone4-sulphonate (NQS; Aldrich Chemical Co., St. Louis, Mo, USA) was prepared by dissolving $250 \mathrm{mg}$ in $50 \mathrm{~mL}$ distilled water. The solution was freshly prepared and protected from light during use. Clark and Lubs buffer solution of $\mathrm{pH} 9$ was prepared by mixing $50 \mathrm{~mL}$ of $0.2 \mathrm{M}$ aqueous solution of boric acid and potassium chloride (1 liter contains $12.368 \mathrm{~g}$ of boric acid and $14.90 \mathrm{~g}$ of potassium chloride) with $21.3 \mathrm{~mL}$ of $0.2 \mathrm{M}$ sodium hydroxide in $200 \mathrm{~mL}$ standard flask [27] and adjusted by $\mathrm{pH}$ meter. Seroxate tablets (SmithKline Beecham Pharmaceuticals, Brentford, $\mathrm{UK}$ ) are labeled to contain $20 \mathrm{mg}$ paroxetine $\mathrm{HCl}$ per tablet. Double distilled water was obtained through WSC-85 water purification system (Hamilton Laboratory Glass Ltd., Ky, USA) and used throughout the work. All solvents and materials used throughout this study were of analytical grade.

\subsection{Preparation of Standard and Sample Solutions}

2.3.1. Paroxetine Hydrochloride (PRX) Standard Solution. An accurately weighed amount (50 mg) of PRX was quantitatively transferred into a $25 \mathrm{~mL}$ calibrated flask, dissolved in $20 \mathrm{~mL}$ distilled water, completed to volume with the same solvent to obtain a stock solution of $2 \mathrm{mg} \mathrm{mL}^{-1}$. This stock solution was further diluted with water to obtain working solutions in the range of $10-80 \mu \mathrm{g} \mathrm{mL}^{-1}$.

2.3.2. Tablets Sample Solution. Twenty tablets were weighed and finely powdered. An accurately weighed quantity of the powdered tablets equivalent to $100 \mathrm{mg}$ of PRX was transferred into a $100 \mathrm{~mL}$ calibrated flask and dissolved in about $40 \mathrm{~mL}$ of distilled water. The contents of the flask were swirled, sonicated for 5 minutes, and then completed to volume with water. The contents were mixed well and filtered rejecting the first portion of the filtrate. The prepared solution was diluted quantitatively with distilled water to obtain a suitable concentration for the analysis.

2.4. General Recommended Procedure. Accurately measured aliquots of PRX solution containing $10-50 \mu \mathrm{g} \mathrm{mL}^{-1}$ were transferred into separate $10 \mathrm{~mL}$ calibrated flasks. One milliliter of Clark and Lubs buffer solution ( $\mathrm{pH}$ 9) was added followed by $1 \mathrm{~mL}$ of NQS solution $(0.5 \%$, w/v). The reaction solution was allowed to proceed at room temperature (25 $\pm 5^{\circ} \mathrm{C}$ ) for 10 minutes. The reaction mixture was completed to volume with methanol, and the resulting solution was measured at $488 \mathrm{~nm}$ against reagent blank treated similarly.

\subsection{Determination of the Stoichiometric Ratio of the Reaction}

2.5.1. Job's Method. Job's method of continuous variation [28] was employed. Master equimolar $\left(5 \times 10^{-3} \mathrm{M}\right)$ aqueous solutions of PRX and NQS were prepared. Series of $10 \mathrm{~mL}$ portions of the master solutions of PRX and NQS were made up comprising different complementary proportions $(0: 10$, $1: 9, \ldots, 9: 1,10: 0$, inclusive) in $10 \mathrm{~mL}$ calibrated flasks containing $1 \mathrm{~mL}$ of buffer solution ( $\mathrm{pH}$ 9). The solution was manipulated as described under the general recommended procedures, Section 2.4.

2.5.2. Limiting Logarithmic Method. In the limiting logarithmic method [29], two sets of experiments were carried out employing the general recommended procedures described above. The first set of experiments was carried out using increasing NQS concentrations $\left(1.9 \times 10^{-3}-9.6 \times 10^{-3} \mathrm{M}\right)$ at fixed PRX concentration $\left(1.37 \times 10^{-5} \mathrm{M}\right)$. The second set of experiments was carried out using increasing PRX concentrations $\left(0.3 \times 10^{-5}-2.04 \times 10^{-5} \mathrm{M}\right)$ at fixed NQS concentration $\left(1.92 \times 10^{-2} \mathrm{M}\right)$. The logarithms of the obtained absorbances were plotted as function of the logarithms of the NQS and PRX concentration in the first and second sets of experiments, respectively. The slopes of the fitting lines in both sets of experiments were calculated. 


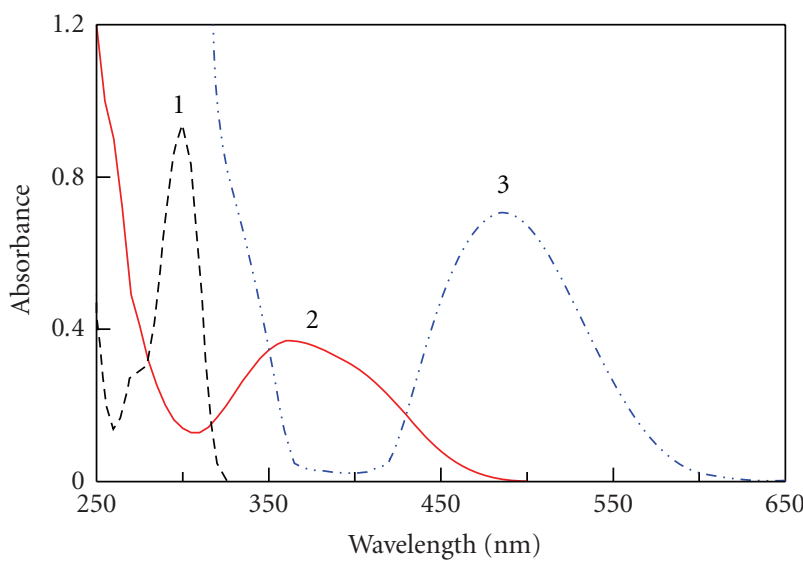

Figure 1: (1) Absorption spectra of PRX against water, (2) NQS against water, and (3) their reaction product against reagent blank.

\section{Results and Discussion}

3.1. Absorption Spectra. According to the procedure, the absorption spectrum of the product produced by the reaction between PRX and NQS was recorded (Figure 1). The product was orange-colored exhibiting a maximum absorption peak $\left(\lambda_{\max }\right)$ at $488 \mathrm{~nm}$, and the $\lambda_{\max }$ of NQS was $360 \mathrm{~nm}$. The $\lambda_{\max }$ of the product was red-shifted by $248 \mathrm{~nm}$ from the $\lambda_{\max }$ of RRX $(240 \mathrm{~nm})$. In order to eliminate the interference, the measurements were carried out at $488 \mathrm{~nm}$ against the reagent blank.

\subsection{Optimization of Reaction Variables}

3.2.1. Effect of NQS Concentration. The studying of NQS concentrations revealed that the reaction was dependent on NQS reagent (Figure 2). The absorbance of the reaction solution increased as the NQS concentration increased, and the highest absorption intensity was attained at NQS concentration of $0.25 \%(\mathrm{w} / \mathrm{v})$. Higher NQS concentrations up to $1.25 \%$ had no effect on the absorption values. Further experiments were carried out using $0.5 \%$.

3.2.2. Effect of Alkalinity and $p H$. To generate the nucleophile from PRX and activate the nucleophilic substitution reaction, alkaline medium was necessary. Different inorganic bases were tested: sodium hydroxide, disodium hydrogen phosphate, and sodium bicarbonate, all prepared as aqueous solution of a concentration range of $1-25 \times 10^{-3} \mathrm{M}$. Best results were obtained in case of sodium hydroxide where with other bases either precipitation of white colloid occurred upon diluting the reaction solution with organic solvent, high blank readings, nonreproducible results, and/or weak sensitivity were observed. Studies for optimization of sodium hydroxide concentration revealed that the optimum concentration was $2-25 \times 10^{-3} \mathrm{M}$ (Figure 2). As well, it was found that the use of alkaline buffer solution gives more precise readings over the use of $\mathrm{NaOH}$. In a separate series of experiments, the influence of $\mathrm{pH}$ on the absorbance of PRX-NQS product was investigated. The results revealed

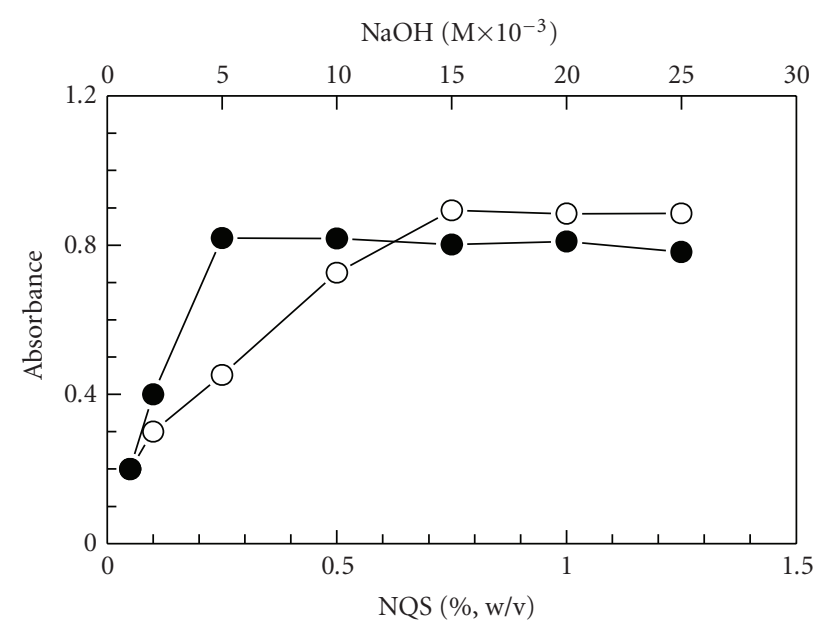

FIGURE 2: Effect of NQS (•) and $\mathrm{NaOH}(\circ)$ concentrations on the reaction of PRX with NQS. PRX $\left(40 \mu \mathrm{g} \mathrm{mL}^{-1}\right): 1 \mathrm{~mL} ; \mathrm{NaOH}: 1 \mathrm{~mL}$; NQS: $1 \mathrm{~mL}$; temperature: $25 \pm 5^{\circ} \mathrm{C}$; reaction time: 10 minutes.

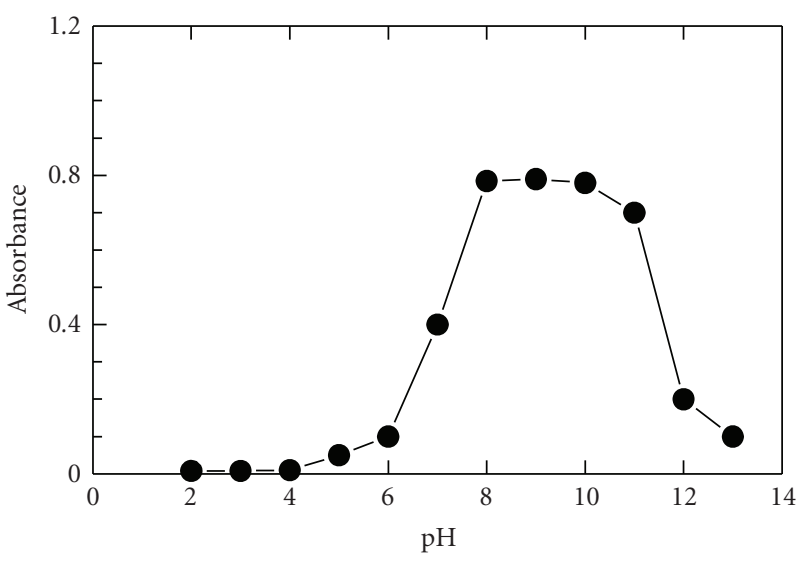

FIgure 3: Effect of $\mathrm{pH}$ on the reaction of PRX with NQS. PRX $\left(40 \mu \mathrm{g} \mathrm{mL}^{-1}\right): 1 \mathrm{~mL}$; Clark and Lubs buffer solution: $1 \mathrm{~mL}$; NQS $(0.5 \%, \mathrm{w} / \mathrm{v}): 1 \mathrm{~mL}$; temperature: $25 \pm 5^{\circ} \mathrm{C}$; reaction time: 10 minutes.

that the absorbances at $\mathrm{pH}<6$ were close to 0 , indicating that under acidity, PRX has difficulty to react with NQS (Figure 3). This was possibly due to the fact that the amino group (piperazinyl-NH) of PRX exists in the form of hydrochloride amine salt, thus, it loses the nucleophilic substitution capability. At $\mathrm{pH}>6$, the absorbance increased rapidly with the increase in the $\mathrm{pH}$, as the amino group of PRX turns into the free- $\mathrm{NH}$, rather than the $\mathrm{HCl}$ salt, facilitating the nucleophilic substitution reaction. The maximum absorption values were attained in the range of $\mathrm{pH}$ at $8-10$. At $\mathrm{pH}>10$, the absorbance of solution obviously decreased. This was attributed probably to the increase in the amount of hydroxide ion that holds back the condensation reaction between PRX and NQS. In order to keep the high sensibility for determination of PRX, the experiment was carried out at $\mathrm{pH} 9$. 
TABLE 1: Effect of diluting and extracting solvents on the intensity of the reaction product of PRX with NQS.

\begin{tabular}{lccc}
\hline Diluting solvent & Absorbance $^{(\mathrm{a})}$ & Extracting solvent & Absorbance $^{(\mathrm{a})}$ \\
\hline Methanol & 0.802 & Carbon tetrachloride & 0.587 \\
Ethanol & 0.781 & Chloroform & 0.808 \\
Isopropanol & 0.778 & Dichloromethane & 0.716 \\
Acetone & 0.245 & Ethyl acetate & 0.362 \\
Acetonitrile & 0.686 & Toluene & 0.267 \\
Dimethylformamide & 0.346 & Benzene & 0.239 \\
1,4-dioxane & 0.617 & & \\
\hline
\end{tabular}

(a) Values for all solvents are mean of three determinations; the RSDs for the readings were $<3$.

TABLE 2: Recovery studies for determination of PRX by the proposed method.

\begin{tabular}{lccc}
\hline Sample number & PRX & & Recovery $(\% \pm S D)^{(\mathrm{a})}$ \\
\hline 1 & Added $\left.(\mu \mathrm{g} \mathrm{mL})^{-1}\right)$ & Found $(\mu \mathrm{g} \mathrm{mL})^{-1}$ & $99.5 \pm 1.85$ \\
2 & 2.0 & 1.99 & $98.9 \pm 1.20$ \\
3 & 3.0 & 2.97 & $101.3 \pm 0.92$ \\
4 & 4.0 & 4.05 & $97.6 \pm 0.84$ \\
5 & 5.0 & 4.88 & $99.7 \pm 1.50$ \\
\hline
\end{tabular}

${ }^{(a)}$ Values are mean of three determinations.

3.2.3. Effect of Temperature and Time. The effect of temperature on the reaction was studied by carrying out the reaction at different temperatures $\left(25-90^{\circ} \mathrm{C}\right)$. The results (Figure 4) revealed that increasing the temperature had negative effect on the absorption values of the reaction solution. This was probably attributed to the instability of the PRX-NQS derivative. For this reason, further experiments were carried out at room temperature $\left(25 \pm 5^{\circ} \mathrm{C}\right)$. The effect of time on the formation of the reaction product was investigated by carrying out the reaction for different times. The maximum absorbance intensity was attained after 5 minutes, and longer reaction time up to 25 minutes did not affect the absorbance intensity (Figure 4). For more precise results, further experiments were carried out at 10 minutes.

3.2.4. Effect of Organic Solvents. It was found that the PRX-NQS product is insoluble in the aqueous reaction medium. For spectrophotometric measurements, the reaction product might be either dissolved in a miscible organic solvent of lower polarity than water or extracted with an immiscible extractive solvent. Different solvents were tested for dilution: methanol, ethanol, isopropanol, acetone, acetonitrile, dimethylsulphoxide, and 1,4-dioxane. The highest readings were obtained when methanol was used for dilution (Table 1). In a separate series of experiments, different nonmiscible solvents were tested for extraction of the PRX-NQS product: carbon tetrachloride, chloroform, dichloromethane, ethyl acetate, toluene, and benzene. The highest readings were obtained when chloroform was used for extraction. The performance of both extractive and nonextractive procedures (in terms of sensitivity and background readings) was comparable. In order to simplify the analytical procedures, the simple nonextractive procedure

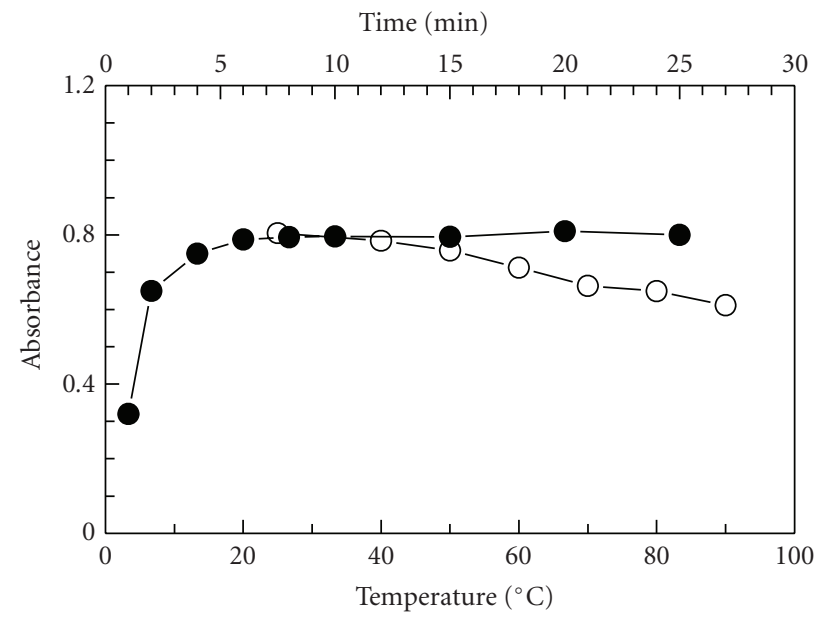

FIGURE 4: Effect of temperature $(\circ)$ and time $(\bullet)$ on the reaction of PRX with NQS. PRX $\left(40 \mu \mathrm{g} \mathrm{mL}^{-1}\right): 1 \mathrm{~mL}$; Clark and Lubs buffer solution ( $\mathrm{pH}$ 9): $1 \mathrm{~mL}$; NQS $(0.5 \%, \mathrm{w} / \mathrm{v}): 1 \mathrm{~mL}$.

(dilution with methanol) was chosen as optimum condition for the further experiments.

3.2.5. Stability of the Chromogen. Under the aforementioned optimum conditions, the reaction between PRX and NQS was completed within 5 minutes at room temperature, and the absorbance no longer changed after standing for up to 25 minutes. The effect of time on the stability of the chromogen was studied by following the absorption intensity of the reaction solution (after dilution) at different time intervals. It was found that the absorbance of the chromogen remains stable for at least 4 hours. This allowed the processing of large 


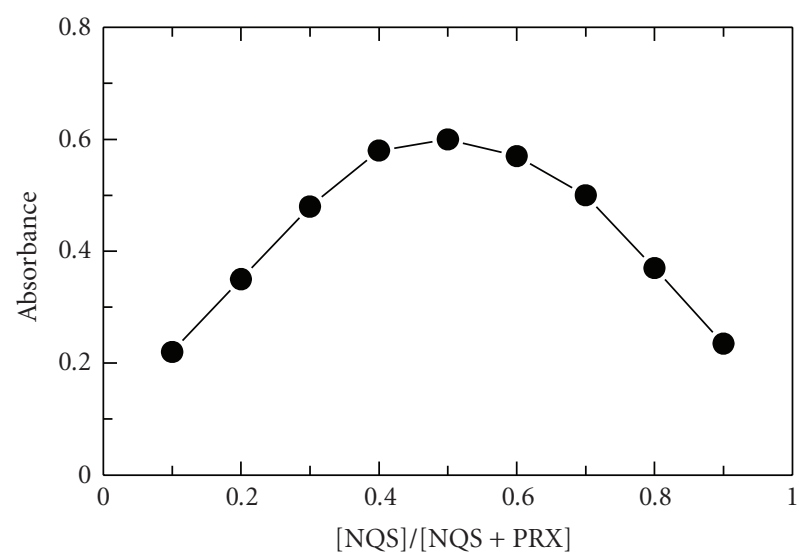

Figure 5: Job's plot for determination of stoichiometry of the reaction between PRX and NQS. [PRX]: $5 \times 10^{-3} \mathrm{M}$; [NQS]: $5 \times$ $10^{-3} \mathrm{M}$; [PRX]+[NQS]: $1 \mathrm{~mL}$; Clark and Lubs buffer solution $(\mathrm{pH}$ 9): $1 \mathrm{~mL}$; temperature: $25 \pm 5^{\circ} \mathrm{C}$; reaction time: 10 minutes.

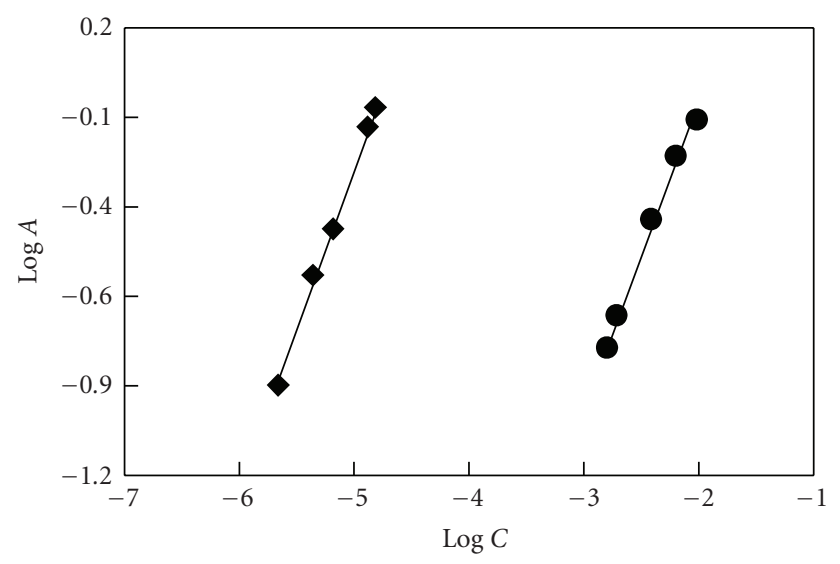

FIGURE 6: Limiting logarithmic plot for molar reactivity of PRX with NQS. $C$ and $A$ are the concentration and absorbance, respectively. For generating the first line $(\checkmark)$, [NQS]: $1.9 \times 10^{-2}$; [PRX]: $0.3 \times 10^{-5}-2.04 \times 10^{-5} \mathrm{M}$; Clark and Lubs buffer solution (pH 9): $1 \mathrm{~mL}$; temperature: $25 \pm 5^{\circ} \mathrm{C}$; reaction time: 10 minutes. For generating the second line $(\bullet),[\mathrm{NQS}]: 1.9 \times 10^{-3}-1.9 \times 10^{-2} \mathrm{M}$; $[\mathrm{PRX}]: 1.37 \times 10^{-5} \mathrm{M}$. The other conditions are the same as those employed in generating the first line.

batches of samples and their comfortable measurements with convenience. This increased the convenience of the methods as well as made it applicable for large number of samples.

3.3. Stoichiometry and Kinetics of the Reaction. Under the optimum conditions, the stoichiometry of the reaction between PRX and NQS was investigated by Job [28] and limiting logarithmic [29] methods. The symmetrical bell shape of Job's plot (Figure 5) indicates that the NQS:PRX ratio was $1: 1$. In the limiting logarithmic method, two straight lines were obtained (Figure 6). The values of the slopes of these lines were 1.0201 and 0.9248 , confirming the $1: 1$ ratio for the reaction. Based on this ratio, and the presence of only one center (piperazinyl $\mathrm{N}-\mathrm{H}$ group) in PRX

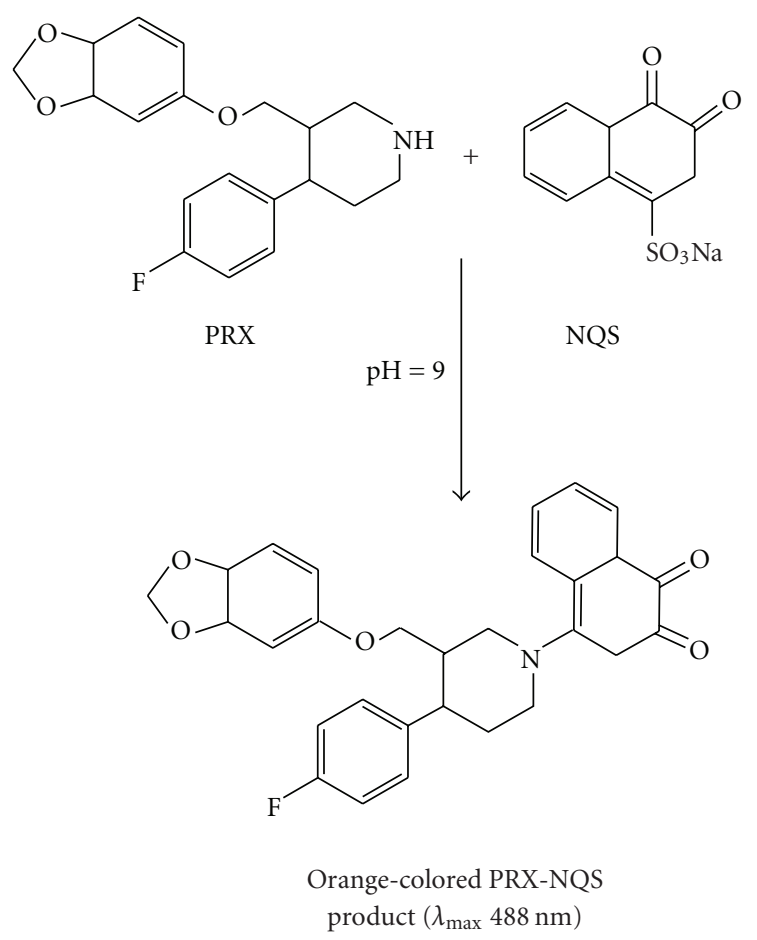

FIgURE 7: Scheme for the reaction pathway of PRX with NQS.

TABLE 3: Analysis of PRX in presence of common excipients by the proposed method.

\begin{tabular}{lc}
\hline Excipient & Recovery $(\% \pm \mathrm{SD})^{(\mathrm{a})}$ \\
\hline Starch $(50)^{(\mathrm{b})}$ & $99.46 \pm 0.46$ \\
Glucose $(10)$ & $99.58 \pm 1.23$ \\
Lactose $(10)$ & $100.37 \pm 0.87$ \\
Acacia $(10)$ & $98.84 \pm 0.39$ \\
Talc (5) & $101.53 \pm 1.02$ \\
MS $^{(\mathrm{c})}(10)$ & $97.97 \pm 1.26$ \\
\hline Average \pm SD & $99.63 \pm 1.23$ \\
\hline
\end{tabular}

(a) Values are mean of three determinations.

(b) Figures in parenthesis are the amounts in mg added per $20 \mathrm{mg}$ of PRX.

(c) MS: Magnesium stearate.

molecule that is available for the substitution reaction, the reaction pathway was postulated to be proceeded as shown in Figure 7.

Under the optimum conditions, the absorbance-time curves for the reaction of PRX at several concentrations (0.3 $\times 10^{-5}-2.04 \times 10^{-5} \mathrm{M}$ ) with a fixed concentration of NQS $\left(1.9 \times 10^{-2} \mathrm{M}\right)$ were generated, and the initial reaction rates $(K)$ were determined from the slopes of the curves. The logarithms of the reaction rates $(\log K)$ were plotted as a function of $\log a r i t h m s$ of PRX concentration $(\log C)$. As seen in Figure 8, a straight line passing through the origin with a slope value of 0.9888 was obtained by fitting the data to the following equation:

$$
\log K=\log K^{\prime}+n \log C,
$$




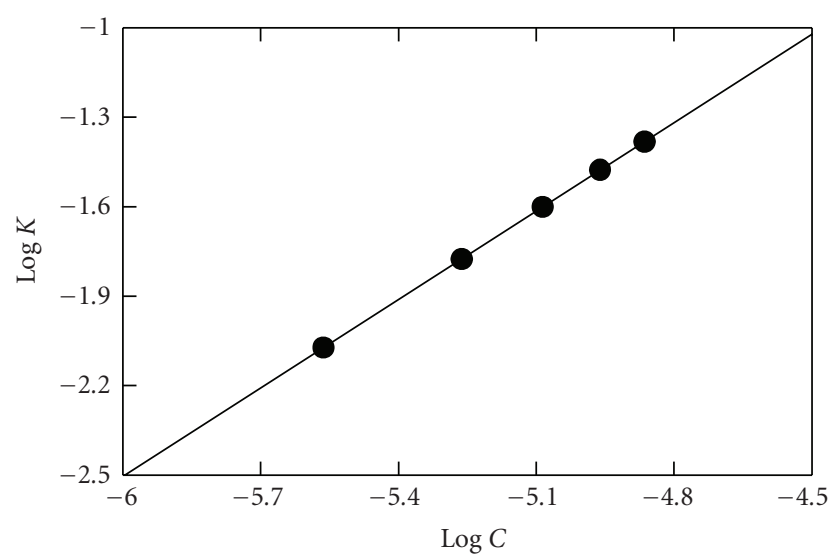

FIGURE 8: Linear plot for $\log C$ versus $\log K$ for the kinetic reaction of PRX with NQS. $C$ is the PRX concentration $\left(0.3 \times 10^{-5}-2.04 \times\right.$ $\left.10^{-5} \mathrm{M}\right)$ and $K$ is the reaction rate $\left(\right.$ second $\left.{ }^{-1}\right)$. Clark and Lubs buffer solution $(\mathrm{pH} 9): 1 \mathrm{~mL}$; NQS $\left(1.9 \times 10^{-2}\right): 1 \mathrm{~mL}$; temperature: $25 \pm$ $5^{\circ} \mathrm{C}$.

where $K$ is reaction rate, $K^{\prime}$ is the rate constant, $C$ is the molar concentration of PRX, and $n$ (slope of regression line) is the order of the reaction. The value of the slope $(\approx 1)$ confirmed that the reaction was first order. However, under the optimized reaction conditions, the concentration of NQS was in much more excess than that of PRX in the reaction solution. Therefore, the reaction was regarded as a pseudofirst-order reaction.

3.4. The Apparent Rate Constant and Activation Energy. The absorbance-time curves at three different temperatures (25, 40 , and $\left.60^{\circ} \mathrm{C}\right)$ were generated using fixed concentrations of PRX $\left(1.7 \times 10^{-5} \mathrm{M}\right)$ and NQS $\left(1.9 \times 10^{-2} \mathrm{M}\right)$. From these curves, the apparent rate constants were calculated. These rates were found to be $6.92 \times 10^{-4}, 6.7 \times 10^{-4}$, and $6.25 \times 10^{-4}$ second $^{-1}$ at 25,40 , and $60^{\circ} \mathrm{C}$, respectively. The activation energy, defined as the minimum kinetic energy that a molecule possess in order to undergo a reaction, was determined using Arrhenius equation [30]:

$$
\log k=\log A-\mathrm{Ea} / 2.303 \mathrm{RT},
$$

where $k$ is the apparent rate constant, $A$ is the frequency factor, Ea is the activation energy, $T$ is the absolute temperature, and $R$ is the gas constant. By plotting log $k^{\prime}$ as a function of $1 / T$, a straight line with a slope value of 1.775 $=-\mathrm{Ea} / 2.303 R$. From this data, the activation energy was found to be $8.12 \mathrm{kcal} \mathrm{mole}^{-1}$. Because of this low activation energy, the nucleophilic substitution reaction between PRX and NQS could be easily taken place, and NQS could be used for determination of PRX.

\subsection{Validation of the Method}

3.5.1. Calibration and Sensitivity. Calibration curve for the determination of PRX by its reaction with NQS was constructed by plotting the absorbances as a function of the corresponding concentrations. The regression equation for
TABLE 4: Influence of small variations in the assay conditions on the analytical performance of the proposed spectrophotometric method for determination of PRX using NQS reagent.

\begin{tabular}{lc}
\hline Parameters & Recovery $(\% \pm \mathrm{SD})^{(\mathrm{a})}$ \\
\hline Recommended conditions $^{(\mathrm{b})}$ & $100.5 \pm 1.35$ \\
NQS concentration $(\%, \mathrm{w} / \mathrm{v})$ & \\
0.25 & $98.8 \pm 0.85$ \\
0.75 & $100.4 \pm 1.25$ \\
Buffer solution $(\mathrm{pH})$ & \\
8.8 & $98.9 \pm 1.87$ \\
9.2 & $101.5 \pm 1.67$ \\
Reaction time $(\mathrm{min})$ & \\
5 & $99.5 \pm 1.87$ \\
15 & $100.2 \pm 0.85$ \\
\hline
\end{tabular}

(a) Values are mean of 3 determinations.

(b) The recommended conditions are given in Section 2.

the results was $A=0.0031+0.1609 C(r=0.9992)$, where $A$ is the absorbance at $488 \mathrm{~nm}, C$ is the concentration of $\mathrm{PRX}$ in $\mu \mathrm{g} \mathrm{mL}^{-1}$ in the range of $1-8 \mu \mathrm{g} \mathrm{mL}^{-1}$, and $r$ is the correlation coefficient. The molar absorptivity $(\varepsilon)$ was $5.9 \times$ $10^{5} \mathrm{~L} \mathrm{~mol}^{-1} \mathrm{~cm}^{-1}$. The limit of detection (LOD) and limit of quantitation (LOQ) were determined using the formula: LOD or LOQ $=\kappa S D a / b$, where $\kappa=3$ for LOD and 10 for LOQ, SDa is the standard deviation of the intercept, and $b$ is the slope. The LOD and LOQ were 0.3 and $0.8 \mu \mathrm{g} \mathrm{mL}^{-1}$, respectively. The precision of the proposed method was determined by analyzing 5 replicate samples of standard PRX solution at one concentration level. The assay gave satisfactory results; the relative standard deviation (RSD) was less than $2 \%$.

3.5.2. Reproducibility. The reproducibility of the proposed method was determined by replicate analysis of five separate solutions of the working standard at three concentration levels of each drug $\left(1.5,3\right.$, and $\left.6 \mu \mathrm{g} \mathrm{mL}^{-1}\right)$. The method gave satisfactory results; RSD did not exceed $2 \%$ indicating the good reproducibility of the proposed method. This precision level is adequate for the precision and routine analysis of the investigated drugs in quality control laboratories.

3.5.3. Accuracy and Interference Liabilities. The accuracy of the proposed method was evaluated by the standard addition method. The recovery values of the added concentrations were $97.6-101.3 \pm 0.84-1.85 \%$ (Table 2 ), indicating the accuracy of the proposed method. Before proceeding with the analysis of PRX in its tablets, interference liabilities were carried out to explore the effect of common excipients that might be added during tablets formulation. Samples were prepared by mixing known amount $(20 \mathrm{mg}$ ) of PRX with various amounts of the common excipients: starch, glucose, lactose, acacia, talc, and magnesium stearate. These laboratory-prepared samples were analyzed by the proposed method applying the general recommended procedure. The recovery values were $97.97-101.53 \pm 0.39-1.26 \%$, with an average recovery of $99.63 \pm 1.23 \%$ (Table 3 ). These data 
TABLE 5: Analysis of PRX-containing tablets by the proposed and the official methods.

\begin{tabular}{|c|c|c|c|c|}
\hline \multirow{2}{*}{ Tablet } & \multicolumn{2}{|c|}{ Recovery $(\% \pm \mathrm{RSD})^{(\mathrm{a})}$} & \multirow{2}{*}{$t$-value $e^{(c)}$} & \multirow{2}{*}{$F$-value ${ }^{(\mathrm{c})}$} \\
\hline & Proposed & Official $^{(\mathrm{b})}$ & & \\
\hline Seroxate tablets & $99.17 \pm 1.06$ & $101.31 \pm 0.48$ & 2.20 & 4.88 \\
\hline
\end{tabular}

confirmed the absence of interference from any of the common excipients with the determination of PRX by the proposed method.

3.5.4. Robustness and Ruggedness. Robustness was examined by evaluating the influence of small variation of method variables including concentration of analytical reagent and reaction time on the performance of the proposed methods. In these experiments, one parameter was changed whereas the others were kept unchanged, and the recovery percentage was calculated each time. It was found that small variation of method variables did not significantly affect the procedures; recovery values were $98.8-101.5 \pm 0.85-1.87 \%$ (Table 4 ). This provided an indication for the reliability of the proposed method during its routine application for the analysis of PRX. Ruggedness was also tested by applying the proposed methods to the assay of PRX using the same operational conditions but using two different instruments at two different laboratories and different elapsed time. Results obtained from lab-to-lab and day-to-day variations were reproducible, as the relative standard deviations (RSDs) did not exceed $2.54 \%$.

3.6. Application of the Proposed Method to Analysis of PRX in Tablets. It is evident from the above-mentioned results that the proposed method gave satisfactory results with PRX in bulk. Thus, its tablets were subjected to the analysis of their PRX contents by the proposed and the official [14] methods. The label-claim percentage was $99.17 \pm 1.06 \%$ (Table 5). This result was compared with that obtained from the official method by statistical analysis with respect to the accuracy (by $t$-test) and precision (by $F$-test). No significant differences were found between the calculated and theoretical values of $t$ - and $F$-tests at $95 \%$ confidence level proving similar accuracy and precision in the determination of PRX by both methods.

\section{Conclusions}

The present study described the successful evaluation of NQS reagent in the development of simple and rapid spectrophotometric method for the accurate determination of PRX in bulk and tablets. In contrast with the previously reported methods for analysis of PRX, the method described herein has many advantages: it does not need expensive sophisticated apparatus, it is simple and rapid, and it has high sensitivity. Furthermore, all the analytical reagents are inexpensive, have excellent shelf life, and are available in any analytical laboratory. Therefore, this method is practical and valuable for its routine application in the analysis of PRX in quality control laboratories.

\section{References}

[1] W. Z. Potter and L. E. Hollister, "Antidepressant agents," in Basic \& Clinical Pharmacology, B. G. Katzung, Ed., pp. 482496, McGraw Hill, New York, NY, USA, 9th edition, 2004.

[2] S. C. Sweetman, Ed., Martindale: The Complete Drug Reference, Pharmaceutical Press, London, UK, 34th edition, 2005.

[3] P. Tucker, R. Zaninelli, R. Yehuda, L. Ruggiero, K. Dillingham, and C. D. Pitts, "Paroxetine in the treatment of chronic posttraumatic stress disorder: results of a placebo-controlled, flexible-dosage trial," Journal of Clinical Psychiatry, vol. 62, no. 11, pp. 860-868, 2001.

[4] L. Iversen and R. A. Glennon, in Burger's Medicinal Chemistry and Drug Discovery, D. J. Abraham, Ed., vol. 6, p. 483, John Wiley \& Sons, New York, NY, USA, 6th edition, 2003.

[5] H. P. A. Nouws, C. Delerue-Matos, A. A. Barros, and J. A. Rodrigues, "Electroanalytical determination of paroxetine in pharmaceuticals," Journal of Pharmaceutical and Biomedical Analysis, vol. 42, no. 3, pp. 341-346, 2006.

[6] N. Erk and J. Biryol, "Voltammetric and HPLC techniques for the determination of paroxetine hydrochloride," Pharmazie, vol. 58, no. 10, pp. 699-704, 2003.

[7] S. Robert, M. Genowefa, and K. Marcin, "Determination of fluoxetine and paroxetine in pharmaceutical formulations by densitometric and videodensitometric TLC," Journal of Planar Chromatography. Modern TLC, vol. 16, no. 1, pp. 19-22, 2003.

[8] A. Venkatachalam and V. S. Chatterjee, "Stability-indicating high performance thin layer chromatography determination of Paroxetine hydrochloride in bulk drug and pharmaceutical formulations," Analytica Chimica Acta, vol. 598, no. 2, pp. 312-317, 2007.

[9] I. A. Zainaghi, V. L. Lanchote, and R. H. C. Queiroz, "Determination of paroxetine in geriatric depression by high-performance liquid chromatography," Pharmacological Research, vol. 48, no. 2, pp. 217-221, 2003.

[10] Z. Zhu and L. Neirinck, "High-performance liquid chromatography-mass spectrometry method for the determination of paroxetine in human plasma," Journal of Chromatography B, vol. 780, no. 2, pp. 295-300, 2002.

[11] P. Massaroti, N. M. Cassiano, L. F. Duarte, et al., "Validation of a selective method for determination of paroxetine in human plasma by LC-MS/MS," Journal of Pharmacy and Pharmaceutical Sciences, vol. 8, no. 2, pp. 340-347, 2005.

[12] O. H. Jhee, H. K. Seo, M. H. Lee, et al., "Determination of paroxetine in plasma by liquid chromatography coupled to tandem mass spectrometry for pharmacokinetic and bioequivalence studie," Arzneimittel-Forschung, vol. 57, no. 7, pp. 455-461, 2007.

[13] British Pharmacopoeia 2003, The Stationary Office, London, UK, 2003. 
[14] United States Pharmacopeial Convention, The United States Pharmacopeia 31, The National Formulary 26, United States Pharmacopeia, Rockville, Md, USA, 2008.

[15] C. B. Eap, G. Bouchoux, M. Amey, N. Cochard, L. Savary, and P. Baumann, "Simultaneous determination of human plasma levels of citalopram, paroxetine, sertraline, and their metabolites by gas chromatography-mass spectrometry," Journal of Chromatographic Science, vol. 36, no. 7, pp. 365-371, 1998.

[16] J. L. Hans, W. Werner, and F. Günter, "Improved sample preparation for the quantitative analysis of paroxetine in human plasma by stable isotope dilution negative ion chemical ionisation gas chromatography-mass spectrometry," Journal of Chromatography B, vol. 779, no. 2, pp. 353-357, 2002.

[17] L. Chien, S. G. Emily, H. K. Sidney, N. Alan, T. C. Ronald, and B. B. Glen, "Determination of paroxetine levels in human plasma using gas chromatography with electroncapture detection," Journal of Chromatography B, vol. 749, no. 2, pp. 275-279, 2000.

[18] L. Labat, M. Deveaux, P. Dallet, and J. P. Dubost, "Separation of new antidepressants and their metabolites by micellar electrokinetic capillary chromatography," Journal of Chromatography B, vol. 773, no. 1, pp. 17-23, 2002.

[19] A. Onal, S. E. Kepekçi, and A. Oztunç, "Spectrophotometric methods for the determination of the antidepressant drug paroxetine hydrochloride in tablets," Journal of AOAC International, vol. 88, no. 2, pp. 490-495, 2005.

[20] I. A. Darwish and I. H. Refaat, "Spectrophotometric analysis of selective serotonin reuptake inhibitors based on formation of charge-transfer complexes with tetracyanoquinodimethane and chloranilic acid," Journal of AOAC International, vol. 89, no. 2, pp. 326-333, 2006.

[21] I. A. Darwish, "Development and validation of spectrophotometric methods for determination of fluoxetine, sertraline, and paroxetine in pharmaceutical dosage forms," Journal of AOAC International, vol. 88, no. 1, pp. 38-45, 2005.

[22] Q.-M. Li, J. Li, and Z.-J. Yang, "Study of the sensitization of tetradecyl benzyl dimethyl ammonium chloride for spectrophotometric determination of dopamine hydrochloride using sodium 1,2-naphthoquinone-4-sulfonate as the chemical derivative chromogenic reagent," Analytica Chimica Acta, vol. 583, no. 1, pp. 147-152, 2007.

[23] M. Hasani, L. Yaghoubi, and H. Abdollahi, "A kinetic spectrophotometric method for simultaneous determination of glycine and lysine by artificial neural networks," Analytical Biochemistry, vol. 365, no. 1, pp. 74-81, 2007.

[24] Q.-M. Li and Z.-J. Yang, "Spectrophotometric determination of aminomethylbenzoic acid using sodium 1,2naphthoquinone-4-sulfonate as the chemical derivative chromogenic reagent," Spectrochimica Acta Part A, vol. 66, no. 3, pp. 656-661, 2007.

[25] I. A. Darwish, "Kinetic spectrophotometric methods for determination of trimetazidine dihydrochloride," Analytica Chimica Acta, vol. 551, no. 1-2, pp. 222-231, 2005.

[26] L. Xu, H. Wang, and Y. Xiao, "Spectrophotometric determination of ampicillin sodium in pharmaceutical products using sodium 1,2-naphthoquinone-4-sulfonic as the chromogentic reagent," Spectrochimica Acta Part A, vol. 60, no. 13, pp. 30073012, 2004.

[27] M. Pesez and J. Bartos, in Colorimetric and Fluorimetric Analysis of Organic Compounds and Drugs, pp. 628-630, Marcel Dekker, New York, NY, USA, 1974.

[28] P. Job, "Analytica Chimica Acta 16 (1936)," in Advanced Physicochemical Experiments, p. 54, Oliner and Boyd, Edinburgh, UK, 2nd edition, 1964.
[29] J. Rose, Advanced Physicochemical Experiments, Pitman, London, UK, 1964.

[30] A. Martin, J. Swarbrick, A. Cammarata, and A. Chun, Physical Pharmacy: Physical Chemical Principles in the Pharmaceutical Sciences, Lea \& Febiger, Philadelphia, Pa, USA, 3rd edition, 1983. 


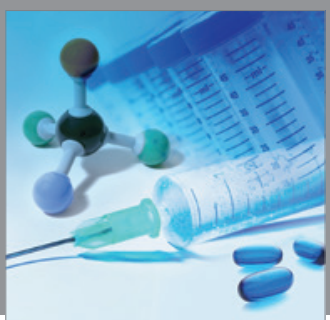

International Journal of

Medicinal Chemistry

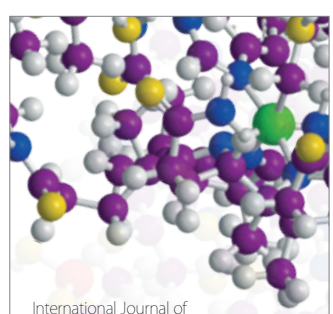

Carbohydrate Chemistry

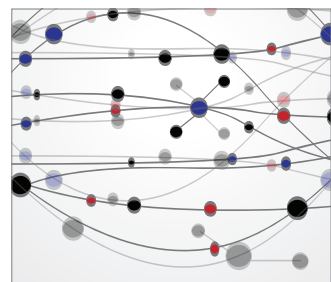

The Scientific World Journal
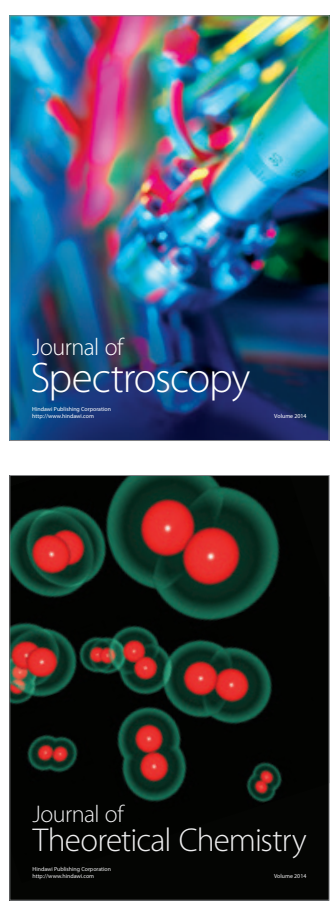
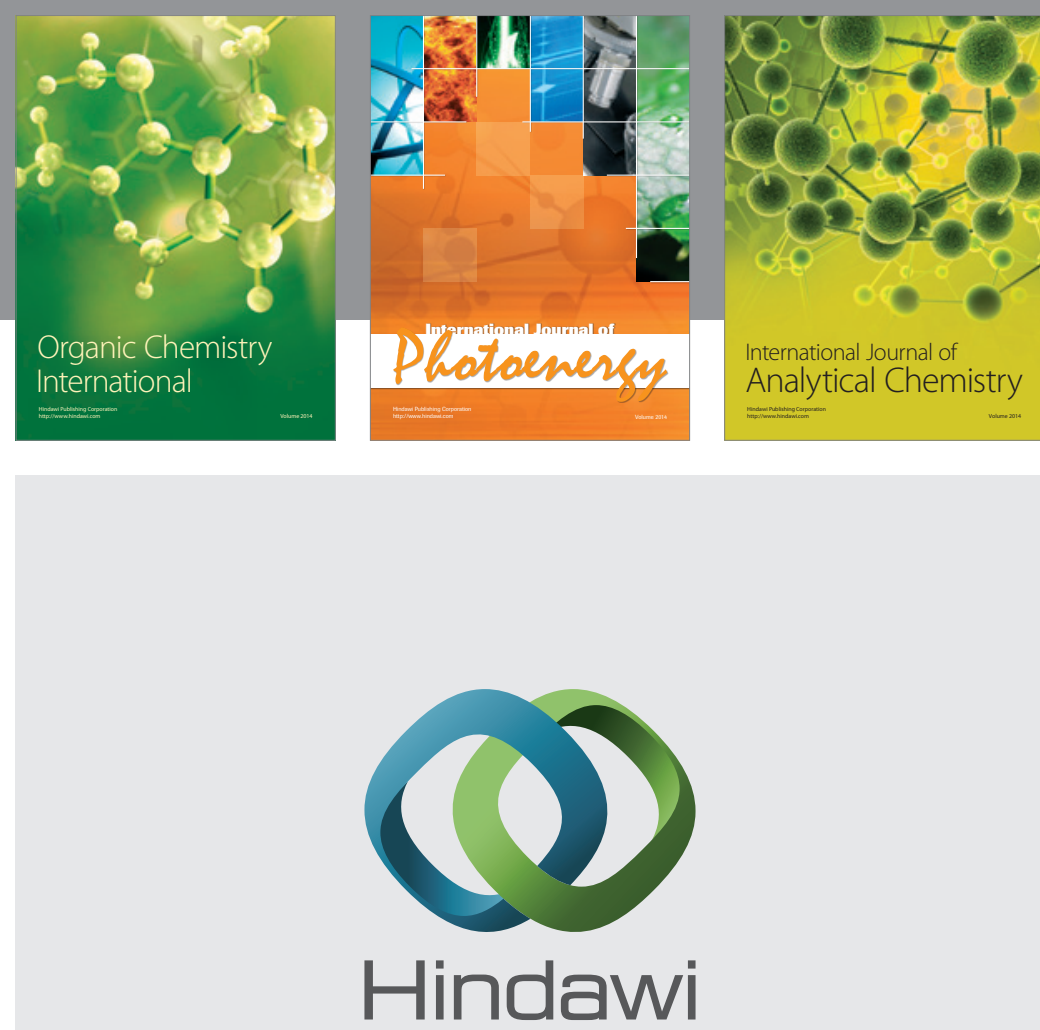

Submit your manuscripts at

http://www.hindawi.com
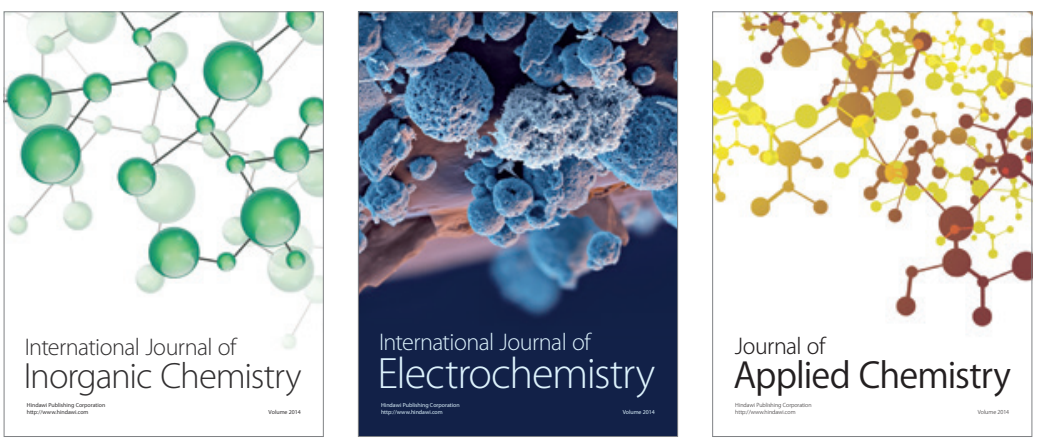

Journal of

Applied Chemistry
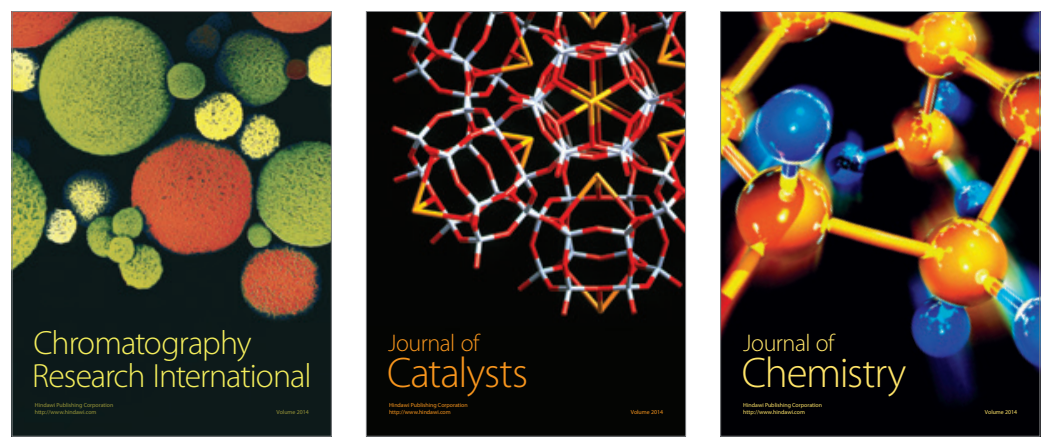
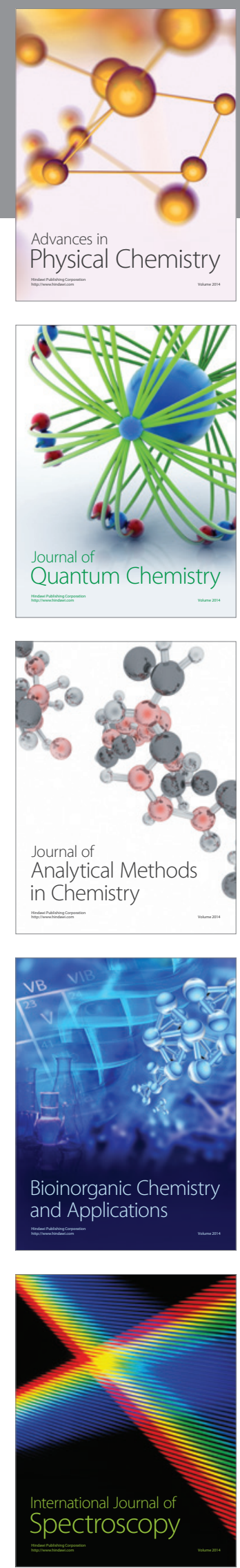